\title{
Utilizing combinations of new approaches to peptide and peptidomimetic design for G-Protein Coupled Receptors
}

\author{
Victor J. Hruby, Minying Cai, Yanh Zhou, Jose Rios-Monterrosa \\ Departmentof Chemistry and Biochemistry, University of Arizona, Tucson, Arizona, United States
}

https://doi.org/10.17952/35EPS.2018.050

Living systems are very smart chemists and about 700 million years ago when animal life started most small molecule synthesis was discontinued. Now we get most of our small molecules from our diet. Instead, animal life evolved much more robust nucleic acid, peptide and protein, sugar and lipid chemistries. Not surprisingly, most of our current small molecule drugs have toxicities and mostly treat symptoms of our degenerative diseases. Drugs for the future need to be composed from peptides, proteins, nucleic acids, sugars and lipids to minimize toxicities. Here we will briefly illustrate this approach utilizing the melanocortin system (five receptors) and its native precursor peptide ligands derived from proopiomelanocorin (POMC), a primordial animal system involved in most of the biological activities, critical to our survival and good health.

The melanocortin system is composed of five receptors (MC1R, MC2R, MC3R, MC4R and MC5R) and are found throughout the body and brain. They are critical for most of the major biological functions critical for survival and good health including pigmentation, response to stress, feeding behavior, sexual behavior, immune response, inflammatory response, cardiovascular and kidney function and many others, some still being discovered. The endogenous peptide ligands for these receptors are derived from a single primordial gene POMC and include ACTH (specific for MC2R), $\alpha$-, $\beta$ - and $\gamma$-MSHs which interact with MC1R, MC3R, MC4R and MC5R, but they are nonselective and have very short half lives in vivo (a few minutes) and all are agonists. A major goal in this research is to develop novel, potent, receptor selective agonists and antagonists (orthosteric and allosteric) that are selective for these receptors and are more stable and bioavailable, and that can (or cannot) cross the blood brain barrier (1). Here we will briefly discuss our recent efforts toward these goals, using the full repertoire of approaches to peptide and peptidomimetic design we have developed over the past 40 years $(2,3)$. These include computer based drug design, biophysical methods, conformational constraints, novel amino acids, novel cyclic systems, $\mathrm{N}$-methylation and peptide mimetic design.

In earlier studies, we designed a number of peptide analogues of $\alpha$-MSH using these methods including [Nle ${ }^{4}$, D-Phe $\left.{ }^{7}\right] \alpha-M S H\left(M T-I\right.$, NDP- $\alpha$-MSH) (Ac-Ser-Tyr-Ser-Nle-Glu-His-DPhe-Arg-Trp-Gly-Lys-Pro-Val-NH ${ }_{2}$, MTII-(Ac-Nle-c[Asp-DPhe-Arg-Trp-Lys]- $\mathrm{NH}_{2}$ ) and Shu-9119-(Ac-Nle-c[Asp-His-D-Nal(2')-Arg-Trp-Lys]-NH 2 . These are highly potent (nanomolar to subnanomolar), stable (2 hours to 2 days) and bioavailable. MT-II crosses the blood brain barrier, MT-I does not. Neither are receptor selective except that neither interacts with the MC2R. SHU-9119 is very unique being a nanomolar agonist at the MC1R and the MC5R and a potent antagonist at the MC3R and MC4R. These ligands have been widely used by us and worldwide to make many of the biological discoveries for the system including biological activities in the brain.

An example of a novel allosteric cyclopeptidomimetic we replaced the arginine residue in a cyclic disulfide analogue of MT-II with a guanindinylbutyl group at various backbone locations to give for example Ac-c[CysHis-D-Phe-Cys-guanidinylbutyl-Cys]-Trp- $\mathrm{NH}_{2}$ which was a potent $\left(\mathrm{IC}_{50}=1.8 \mathrm{nM}\right)$, highly selective, MC1R antagonist (4).

In an extensive collaboration with Professor Horst Kessler and his group (5), we examined a very comprehensive library of backbone N-methylated cyclic lactam analogues related to MT-II. A number of highly selective ligands were obtained, the selectivity depending exclusively on the backbone $\mathrm{N}$-methylation. For example, the Ac-Nlec[Asp-N-MeHis-D-Phe-NMeArg-N-MeTrp-NMeLys]- $\mathrm{NH}_{2}$ ligand was a potent $\left(\mathrm{IC}_{50}, 14 \mathrm{nM}\right)$ agonist for the MC1R. Comprehensive NMR studies were done to examine the conformational effects of N-methylation on the biological activity profile which has been used to further design novel constrained ligands for the melanocortin receptors.

In an effort to obtain nonpeptide peptidomimeticligands, we have prepared a number of designed novel bicyclic heterocyclic ligands such as 7 in Figure 1. Surprisingly, very few such bicyclic heterocycles have been designed and published. Compound 1 was found to be a very selective and potent $\left(\mathrm{IC}_{50}=0.4 \mathrm{nM}\right)$ at the $\mathrm{MC} 5 \mathrm{R}$, while its 
enantiomer was inactive at the MC5R (6).

A combination of site specific mutagenesis, domain swapping, computational chemistry, and computer assisted docking of receptor selective ligands at the various melanocortin receptors has been developed for this project. These experiments have provided three dimensional receptor models for the 5 melanocortin receptors which distinguish different critical residues for binding of agonist and antagonist ligands at these receptors. One approach to testing the validity of these models for use in computer assisted drug design is to test these models by designing a melanocortin 1 receptor agonist ligand using only eukaryotic $\alpha$-amino acids. This led us (7) to the design of $\left[\mathrm{Leu}^{3}, \mathrm{Leu}^{7}, \mathrm{Phe}^{8}\right]-\alpha-\mathrm{MSH}-\mathrm{NH}_{2}$ which was found to be a highly MC1R selective agonist for the hMC1R, both in vitro and in vivo .

The above and many other studies have provided us with the excellent structural tools for agonist and antagonist design for 4 human melanocortin receptors. This provides us with the potential of applying these tools for a variety of medical needs, including pigmentation without sun for the prevention of melanoma cancer and other pigmentary disorders, for treatment of melanoma cancer, for treatment of stress related disorders, for feeding behavior disorders, for sexual behavior and dysfunction, for neurodegenerative diseases, immune response disorders and many others. These are all major current goals.

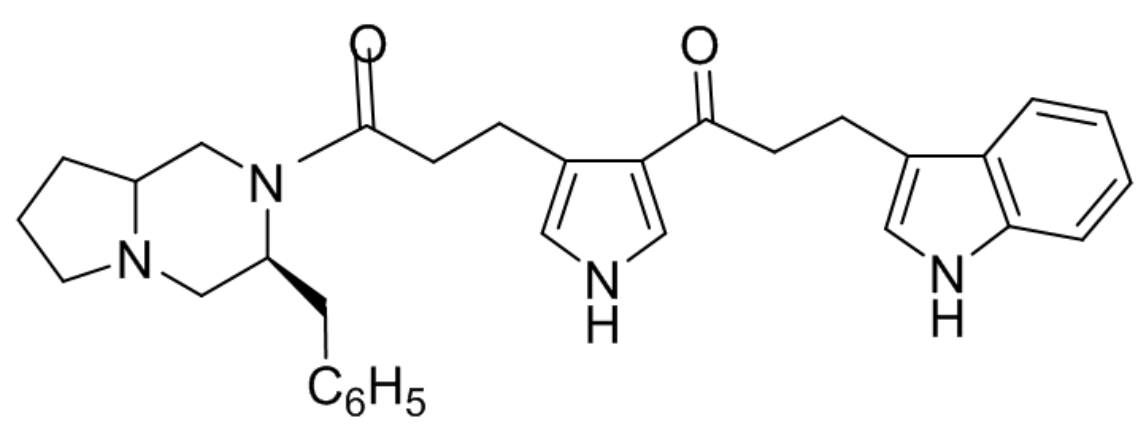

Figure 1: Structure of Heterocyclic Ligand for Melanocortin Receptors

\section{Acknowledgments}

We thank the U.S. Public Health Service, National Institutes of Health and Tech Launch Arizona for partial support of these studies, and to the many undergraduate, graduate, and postdoctoral students who have participated in this research and especially Professor Mac Hadley (deceased) who collaborated with us for over 40 years on this research.

\section{References}

1. Cai, M., and Hruby, V.J., CurrentProtein and Peptide Science, 17 (2016) 488.

2. Hruby, V.J., Nature Rev. Drug Discov., 1 (2002) 847.

3. Hruby, V.J. and Cai, M., Ann. Rev. Pharmacol. and Toxicol., 53 (2012) 557.

4. Ying, J., Gu, X., Cai, M., Dedek, M., Vagner, J., Trivedi, D., and Hruby, V.J., J. Med. Chem., 49 (2006) 6888.

5. Doedens, L., Opperer, F., Cai, M., Beck, J.G., Dedek, M., Palmer, E., Hruby, V.J. and Kessler, H., J. AM. Chem. Soc., 132 (2010) 8115.

6. Cain, J.P., Mayorov, A.V., Cai, M., Wang, H., Tan, B., Chandler, K., Lee, Y.S., Petrov, R.R., Trivedi, D. and Hruby, V.J., Bioorg. Med. Chem. Letts., 16 (2006) 5462.

7. Zhou, Y., Haghighi, S.M., Zoi, I., Sawyer, J.R., Hruby, V.J. and Cai, M., J. Med. Chem., 60 (2017) 9320. 